OPEN ACCESS

Edited by: Sébastien Roger, Université de Tours, France

Reviewed by: Irene Frischauf, Johannes Kepler University of Linz, Austria

Heike Wulff,

University of California, Davis,

United States

${ }^{*}$ Correspondence:

Raphael Rapetti-Mauss Raphael.RAPETTI-MAUSS@univcotedazur.fr

Specialty section:

This article was submitted to Pharmacology of lon Channels and Channelopathies, a section of the journal

Frontiers in Pharmacology

Received: 07 January 2020

Accepted: 31 August 2020 Published: 29 September 2020

Citation:

Rapetti-Mauss R, Berenguier C, Allegrini B and Soriani O (2020) Interplay Between Ion Channels and the Wnt/ $\beta$-Catenin Signaling Pathway in Cancers.

Front. Pharmacol. 11:525020. doi: 10.3389/fphar.2020.525020

\section{Interplay Between Ion Channels and the Wnt/ $\beta$-Catenin Signaling Pathway in Cancers}

\author{
Raphael Rapetti-Mauss *, Camille Berenguier, Benoit Allegrini and Olivier Soriani \\ Inserm, CNRS, iBV, Université Côte d'Azur, Nice, France
}

Increasing evidence point out the important roles of ion channels in the physiopathology of cancers, so that these proteins are now considered as potential new therapeutic targets and biomarkers in this disease. Indeed, ion channels have been largely described to participate in many hallmarks of cancers such as migration, invasion, proliferation, angiogenesis, and resistance to apoptosis. At the molecular level, the development of cancers is characterised by alterations in transduction pathways that control cell behaviors. However, the interactions between ion channels and cancer-related signaling pathways are poorly understood so far. Nevertheless, a limited number of reports have recently addressed this important issue, especially regarding the interaction between ion channels and one of the main driving forces for cancer development: the $W n t / \beta$-catenin signaling pathway. In this review, we propose to explore and discuss the current knowledge regarding the interplay between ion channels and the $\mathrm{Wnt} / \beta$-catenin signaling pathway in cancers.

Keywords: signaling pathways, drug targets, KCNQ1 channel, P2X7, cystic fibrosis transmembrane conductance regulator, hERG, transient receptor protein channels, CIC-2 channel

\section{INTRODUCTION}

Despite decades of intensive research on its physiopathology, cancer remains a leading cause of death worldwide according to the World Health Organisation. Traditional cancer treatments including radiation therapy, chemotherapy, and surgery have substantially improved patients' conditions. However, several challenges in curing cancer remain in front of us. Side effects generated by cancer treatments significantly impact patients quality of life; high patients intrinsic heterogeneity revealed treatments resistance, and finally the efficiency of treatment used for some types of cancer remains very low such as for pancreatic cancer. These circumstances highlight the urgent need to identify new therapeutic strategies in treating cancers.

Ion channels are transmembrane proteins allowing ions to cross biological membranes thereby generating electrical current and variation in membrane potential. Ion channels are classified in five main families, Potassium channels, Chloride channels, Sodium channels, Calcium channels that are selective for their specific ion, and non-selective ion channels that allow the passage of several species of ions. The functions attributed to ion channels were first confined to cell excitability and regulation of hydro-electrolytic balance. Interestingly, since the 1980's ion channels have also been described to be involved in controlling processes regulating cell behaviors such as proliferation, 
migration, invasion and differentiation. In 1984, the pioneer work of DeCoursey describing the involvement of potassium channels in the proliferation of T lymphocytes (DeCoursey et al., 1984) opened new perspectives in studying the biology of ion channels. The first evidence for such functions of ion channels in cancer cells emerged in the late 1980's with studies describing that the pharmacological inhibition of ion channels reduces tumor cells proliferation (Yamashita et al., 1987; Lee et al., 1988; Pancrazio et al., 1989; Taylor and Simpson, 1992). Since then, the field has greatly expanded, and it has been shown that ion channels are involved in virtually all the hallmarks of cancers described by Hanahan et al. (Prevarskaya et al., 2010; Prevarskaya et al., 2018). Although the involvement of ion channels in cancer has been clearly described, how ion channels interact with cancer-related signaling pathways from a mechanistic point of view, and how the expression of ion channels is regulated in cancers is poorly understood. However, this issue has recently emerged in the literature and a growing number of reports tackle these questions. Cancer initiation and development is often associated to altered signaling pathway involved in cell and tissue homeostasis (Sanchez-Vega et al., 2018). Among these signaling pathways, the $\mathrm{Wnt} / \beta$-catenin pathway emerged as the most associated pathway to ion channels in the literature. This review aims to summarize the current knowledge regarding the interplay between ion channels and the $\mathrm{Wnt} / \beta$-catenin signaling pathway.

\section{THE WNT SIGNALING PATHWAY}

Since the first description of Wingless Int 1 (Wnt1) gene in virally induced breast tumors (Nusse and Varmus, 1982), the Wnt signaling pathway has been subjected to intense research, ranging from development to cancer. Its role in morphogenesis and adult stem cell renewal is closely linked to its involvement in cancer development (Nusse and Clevers, 2017). Mechanistically, Wnt signaling pathway can take mostly two directions: the canonical (Wnt/ $\beta$-catenin), influencing cell-fate, proliferation, as well as self-renewal and the non-canonical ( $\beta$-cateninindependent), Wnt/PCP, Wnt/Ca ${ }^{++}$, Wnt/STOP, influencing planar polarity (Tree et al., 2002), $\mathrm{Ca}^{2+}$ homeostasis (Sheldahl et al., 2003), or protein stabilization (Davidson et al., 2009), respectively. Both are involved in cancer but canonical Wnt/ $\beta$ catenin remains the most studied in a large variety of tissues.

In mammals, there are more than 19 Wnt ligands which are expressed in a tissue-specific manner. After their translation into proteins Wnt ligands are modified with attachment of a palmitoleic acid (palmitoylation) by the ER-resident acyltransferase Porcupine (Kadowaki et al., 1996). This modification allows binding of Wnt ligand to the ERtransmembrane protein Evi/Wls, and therefore the transport to Golgi apparatus (Bänziger et al., 2006) assisted by the $\mathrm{p} 24$ protein (Buechling et al., 2011). Wnt ligands are either released by solubilisation (Mulligan et al., 2012), by exosomes (Gross et al., 2012), or in lipoproteins particles (Neumann et al., 2009) or linked to the plasma membrane. Importantly, after being secreted, Wnt ligands can form a gradient and act as directional growth factor, as it is the case in intestinal colonic crypts (Medema and Vermeulen, 2011)

Here we will focus on Wnt canonical pathway, which depends on $\beta$-catenin stability. In the absence of Wnt ligand (Wnt OFF state), cytosolic $\beta$-catenin is sequestrated by a destruction complex (DC) composed of the tumor suppressor Axin acting as scaffold protein, the tumor suppressor Adenomatous Polyposis Coli (APC), the glycogen synthase kinase $3 \beta$ $(\mathrm{GSK} 3 \beta)$ and casein kinase $1 \alpha(\mathrm{CK} 1 \alpha)$. Inside this DC, $\beta$ catenin is bound to Axin and phosphorylated by the two constitutively active kinases GSK $3 \beta$ and CK1 $\alpha$ at specific Serine and Threonine residues (Liu et al., 2002). Then, $\beta$ catenin is ubiquitylated by the F-box containing protein E3 ubiquitin ligase $(\beta$-TrCP) and addressed to the proteasome for degradation (Aberle et al., 1997; Latres et al., 1999).

Initiation of the Wnt canonical ( $\beta$-catenin-dependent) pathway (Wnt ON state) is mediated by engagement of Frizzled $(\mathrm{Fz})$ receptor and LRP5/6 co-receptor with Wnt ligands. Activation of the pathway leads to degradation complex relocation near Fz/LRP complexes, where LRP is phosphorylated by CK1 $\alpha$ and GSK3 $\beta$. The phosphorylation of LRP triggers Dishevelled (Dvl) protein polymerisation, thus inactivating the degradation complex (Metcalfe et al., 2010). Another study suggests a direct inhibition of GSK3 $\beta$ by the phosphorylated-LRP co-receptor (Stamos et al., 2014). Hence, $\beta$ catenin is not degraded, can accumulate in the cytoplasm and translocate into the nucleus. $\beta$-catenin then complexes with lymphoid enhanced factor (LEF) and T-cell factor (TCF) to act as a transcription factor activating target genes (Li et al., 2012). In the Wnt OFF state, TCF interacts with Groucho, preventing transcriptional activation of Wnt target genes (Cavallo et al., 1998). Interestingly, $\beta$-catenin plays a second role in maintaining epithelial junctions, by binding to the cytoplasmic tail of Ecadherin (Peifer et al., 1992). Thus, presence of epithelial junctions is a limiting factor for Wnt/ $\beta$-catenin activity, and reciprocally (Huels et al., 2015).

The $W n t / \beta$-catenin pathway is tightly regulated. For example, the two homologues E3 ubiquitin ligases ZNRF3/RNF43 can induce $\mathrm{Fz}$ receptor lysosomal degradation, thus inhibiting Wnt signaling. R-spondins are secreted proteins, interacting with their receptor LGR4-6, able to inhibit ZNRF3/RNF43 activity (de Lau et al., 2011). Furthermore, Hippo and Wnt pathway have been closely linked, since the Hippo transducer YAP/TAZ plays an integrative role inside the DC, allowing $\beta$-TrCP recruitment to the complex and subsequent $\beta$-catenin degradation (Azzolin et al., 2014). Others regulations are nicely reviewed by Zhan et al. (2017).

As mentioned above, Wnt canonical pathway is widely implicated in cancer diseases. The first mutations have been found in the APC gene, leading to Familiar Adenomatous Polyposis (Kinzler et al., 1991; Nishisho et al., 1991), and now found in the majority of colorectal cancers (Wood et al., 2007). Mutations of Axin1 are present in hepatocellular carcinoma (Satoh et al., 2000). Gain of function $\beta$-catenin mutations are reported in melanoma (Rubinfeld et al., 1997). Loss of function 
mutations in Rnf43 or Znrf3 are found in pancreatic cancer (Wu et al., 2011) and adrenocortical carcinoma (Assié et al., 2014). Wnt signaling has also been implicated in cholangiosarcoma (Chan-On et al., 2013), leukemia (Wang et al., 2010) and in 50\% of breast cancer cases (Lin et al., 2000).

\section{WNT SIGNALING PATHWAY INTERPLAY WITH POTASSIUM CHANNELS}

With more than 80 members, the potassium channel family is the widest group of ion channels. They participate in a multitude of biological processes from cell excitability to water and electrolyte homeostasis. They are especially essential in maintaining membrane resting potential. Increasing evidence point out the involvement of this family of ion channel in the physiopathology of cancers (Huang and Jan, 2014; Pardo and Stühmer, 2014). However, the molecular mechanisms sustaining potassium channels involvement in cancer are still unclear. This section reviews recent work that report the function of potassium channels in the Wnt/ $\beta$-catenin pathway.

\section{KCNQ1}

The potassium channel KCNQ1, also called Kv7.1, belongs to the family of voltage-gated potassium channel. It is constituted by the assembly of four $\alpha$ subunits forming the pore of the channel. This channel can associate with ancillary subunits from the KCNE family which drastically change the channel features regarding the KCNE associated (Bendahhou et al., 2005; Jespersen et al., 2005). Association with KCNE1, shows a delay in KCNQ1 activation and in this case the complex KCNQ1/ KCNE1 also called IKs, is essential to cardiac action potential repolarisation. With KCNE3 and KCNE2, KCNQ1 is no longer voltage-dependent and participates to transepithelial transport in nearly every epithelium. The effect of the last two members of the KCNE family on KCNQ1 are more discussed in the literature, however it appears that KCNE4 and KCNE5 mostly act as inhibitors of KCNQ1 activity (Abbott, 2014).

As aforementioned, KCNQ1 plays a key role in the physiology of epithelial transport. Transport mechanisms involve complex networks of ion channels and transporters, creating and dissipating ionic gradients required for the passage of water, electrolytes, nutrients or other substrates through the epithelium (Barrett and Keely, 2000). Thus, the $\mathrm{Na}^{+} / \mathrm{K}^{+}$ATPase pump activity is the main force for transepithelial transport allowing $\mathrm{Na}^{+}$output against $\mathrm{K}^{+}$entry into the cell. In this context, $\mathrm{K}^{+}$ channels provide two main functions: the maintenance of membrane potential and the basolateral recycling of $\mathrm{K}^{+}$that guarantees the maintenance of electrochemical gradients. In the colon, KCNQ1, associated with its regulatory subunit KCNE3, plays a fundamental role in Leiberkühn's crypts homeostasis by maintaining the electrical driving force required for apical secretion of $\mathrm{Cl}^{-}$and water, and $\mathrm{Na}^{+}$absorption (Preston et al., 2010; Rapetti-Mauss et al., 2013).
Recently, studies found an unexpected link between KCNQ1 and epithelial cancers. In a first study, using mouse models, the authors have shown that the loss of KCNQ1 expression promotes the development of adenocarcinoma in the colon. They also showed that the low expression of KCNQ1 is associated with a poor survival prognosis in patients with Colorectal Cancer (CRC) showing hepatic metastases (Than et al., 2014). In line with these findings, another report has shown that in patients with stage II and III colon cancer, low expression of KCNQ1 is associated with poor survival prognosis. This channel therefore seems to be an interesting prognostic marker for the early stages of the disease as well as for the prediction of recurrences (den Uil et al., 2016). These studies also suggest that KCNQ1 acts as a tumor suppressor in CRC. Another study described the same function of KCNQ1 in Hepatocellular Carcinoma (HCC). They have shown using immunohistochemistry that HCC patients' samples show less KCNQ1 expression compared with normal liver tissues. Also, in patients with HCC, the high expression of KCNQ1 significantly correlates with a better patient's survival rate. Therefore, they reach the same conclusion as in CRC, KCNQ1 acts as a tumor suppressor in HCC (Fan et al., 2018).

It is clearly not trivial to explain the function of KCNQ1 as a tumor suppressor protein in epithelial cancers by its classical role in the physiology of epithelial transport. Therefore, could alternative functions of this channel underlie the observed correlations between KCNQ1 expression and cancers development? We and other have explored this issue to identify the molecular mechanism sustaining the tumor suppressor functions of KCNQ1 in CRC and HCC.

It appears that KCNQ1 expression is associated in CRC and HCC to epithelial cell plasticity (Rapetti-Mauss et al., 2017; Fan et al., 2018). Epithelial-to-Mesenchymal Transition (EMT) is a cellular process by which epithelial cells progressively and reversibly acquire mesenchymal features (Ye and Weinberg, 2015; Pastushenko and Blanpain, 2019). This process has largely been associated to cancer development and progression by allowing loss of epithelial structure and promoting cancer cell migration, invasion and drug resistance. In CRC, the expression of KCNQ1 in a panel of cell lines showing different degrees of EMT is associated with epithelial features. Conversely, the channel's expression is lowered in most mesenchymal cell lines. These data raise the hypothesis of a functional link between KCNQ1 and the cellular process of epithelial-to-mesenchymal transition in cancer cells. Interestingly, in CRC as well as in HCC, the rescue expression of KCNQ1 in cell lines that do not express this channel, restores the expression of epithelial markers such as E-cadherin, lowers the expression of mesenchymal markers such as $\mathrm{N}$-cadherin and restores the ability of cell to form spheroids in 3D culture. Moreover, the molecular silencing of KCNQ1 in epithelial CRC cell line reduces the expression of E-cadherin, increases the expression of $\mathrm{N}$-cadherin and impairs the ability to grow as a spheroid in $3 \mathrm{D}$ culture. Therefore, the expression of KCNQ1 seems to promote epithelial features.

An important event characterising EMT is the dislocation of adherens junctions (AJ) causing the loss of cohesion between adjacent cells and therefore disrupting the epithelial integrity. The 
exploration of intestinal crypts by techniques coupling immunocytochemistry and in-situ immunisation (proximity ligation assay), provides data showing that KCNQ1 is directly associated with the $\beta$-catenin/E-cadherin complex in $\mathrm{AJ}$ in colonocytes. Interestingly, the same association between KCNQ1 and $\beta$-catenin has been established in hepatocyte cell lines. At the functional level, silencing of KCNQ1 expression in colon cell lines produces a disruption of the complex, as well as a loss of the integrity of epithelial architecture, which results in a decrease in epithelial electrical resistance. The study of the phosphorylation state of $\beta$-catenin in cell lines where KCNQ1 has been silenced by shRNA, indicates that KCNQ1 is necessary to retain $\beta$-catenin at the plasma membrane. Beside this observation it has been shown that pharmacological or molecular inhibition of KCNQ1 in CRC and in HCC, leads to a potentiation of the Wnt/ $\beta$-catenin signaling pathway. Indeed, silencing of KCNQ1 in CRC induces the relocalisation of $\beta$-catenin in the cytosol and increases the expression of Wnt/ $\beta$-catenin signaling pathway targets such as Cyclin D1 and C-jun. In HCC overexpression of KCNQ1 reduces Wnt target genes expression and Wnt activity using a Top/fop assay. These results indicate that KCNQ1 restricts the $\mathrm{Wnt} / \beta$-catenin signaling pathway activity, stabilising $\beta$-catenin at the plasma membrane. These results reveal a new role for KCNQ1: in parallel with its function of transepithelial transport regulator, this channel plays a predominant role in maintaining the architectural integrity of the epithelium. These results shed new light on the correlation between the level of KCNQ1 expression and the clinical outcome of patients with CRC, since high levels of channel expression, together with the maintenance of adherens junctions guarantee the sequestration of $\beta$-catenin to the membrane, repression of the $\mathrm{Wnt} / \beta$-catenin pathway, and finally reduces the commitment in the EMT process.

The stabilisation of the $\beta$-catenin/E-cadherin/KCNQ1 complex at the $\mathrm{AJ}$ constitutes an explanation to understand the role of KCNQ1 as a tumor suppressor in CRC and HCC. Would the latter only play an anchoring protein role for the $\beta$-catenin/ E-cadherin complex, or is the KCNQ1-induced hyperpolarising potassium flow important in the stabilisation of the complex? In other words, do membrane potential variations influence $\beta$ catenin localisation and activity? The answer is provided by experiments in which CRC cells expressing KCNQ1 are experimentally depolarised by increasing the extracellular $\mathrm{K}^{+}$ concentration $140 \mathrm{mM} \mathrm{K}^{+}$. This protocol causes a cytosolic accumulation of $\beta$-catenin, suggesting that the depolarisation induced by the silencing of the channel potentiates $\mathrm{Wnt} / \beta$ catenin pathway. These results demonstrate the existence of a link between the electrical signature of the cancer cell and the signaling pathways that control cancer cells features. These data are consistent with a recent study that shows that the K-Ras/ RAF/MAPK signaling pathway is directly controlled by membrane potential (Zhou et al., 2015).

There is only few information in the literature regarding how ion channel expression is regulated in the context of cancers. It has been observed that expression of KCNQ1 is decreased in both CRC and HCC. We have tempted to address this question and we reason that the link between KCNQ1 could be bidirectional. Is KCNQ1 expression repressed by Wnt activity?
When the Wnt/ $\beta$-catenin pathway is experimentally stimulated by pharmacological or molecular ways, we observed a decrease in KCNQ1 expression. We also identify that $\beta$-catenin as well as the TCF-4 transcription factor (member of the TCF/LEF family, which regulates the expression of the target genes of the Wnt pathway), bind directly to KCNQ1 promoter and cause the repression of its expression. These results thus reveal that KCNQ1 channel is a new target gene of Wnt pathway (Rapetti-Mauss et al., 2017).

By unveiling the bidirectional interaction between $\mathrm{Wnt} / \beta$ catenin pathway and KCNQ1, these works reveal new functions of potassium channels in healthy tissue architecture, and their implication in the development of tumors. Thus, the fine understanding of these alternative functions could contribute to the characterisation of new markers, and innovative therapeutic targets. These data suggest that KCNQ1, by sequestering $\beta$-catenin at the $\mathrm{AJ}$, restricts activation of $\mathrm{Wnt}$ signaling pathway and acts as a tumor suppressor in multiple epithelial cancers. The mechanism seems to be quite conserved suggesting a key role of KCNQ1 in epithelial homeostasis.

\section{$\mathrm{KCNH} 2$}

Another potassium channel has recently been associated to the Wnt/ $\beta$-catenin pathway, KCNH2 also called hERG or Kv11.1 (Breuer et al., 2019). This channel is a voltage-gated potassium channel, which role in cancer cells has largely been studied especially by the groups of Arcangeli (Becchetti et al., 2019) and Soriani (Crottès et al., 2016). This channel is associated with $\beta 1-$ integrin when the channel is in its closed state, resulting in an increase of metastasis in breast cancer. Whereas, in the open state, the channel association with $\beta 1$-integrin is decreased leading to a reduction in breast cancer metastasis (Becchetti et al., 2017).

A recent report described that an opener of hERG, the NS1643, reduces metastasis spread of breast tumor in vivo by attenuation of the $\mathrm{Wnt} / \beta$ catenin pathway signaling, suggesting that NS1643 mimics the dissociation between hERG and $\beta 1$ integrin (Breuer et al., 2019). The authors observed that activating hERG channel stabilises $\beta$-catenin at the plasma membrane by inhibiting AKT (T308) and GSK3 $\beta$ (S9) phosphorylation. In this case the cytosolic fraction of $\beta$-catenin decreases favouring the association of $\beta$-catenin with E-cadherin. Therefore, NS1634 decreases Wnt pathway activity. This result suggests that the hyperproliferation induced by the opening of hERG reduces Wnt activity by retaining $\beta$-catenin at the plasma membrane. These data are consistent with the data from Arcangeli's lab showing that the open state of hERG lowers metastasis dissemination in breast cancer.

\section{WNT SIGNALING PATHWAY INTERPLAY WITH CHLORIDE CHANNELS}

Chloride channels regulate a variety of cell processes such as excitability and water and mucus secretion. Another important 
aspect of these channels, especially in cancer, is their role in cell volume regulation and $\mathrm{pH}$ level regulation. As for the other ion channel families, different chloride channels have been described depending on their gating properties: calcium-activated, voltagedependant, ligand-dependant. Although their role in cancer has been under study for the past years, their link with the Wnt/ $\beta$ catenin pathway remains poorly investigated.

\section{CFTR}

Cystic Fibrosis Transmembrane Conductance Regulator (CFTR) is widely expressed in the apical epithelial membranes. CFTR is an anion channel regulating fluid transport and $\mathrm{pH}$ levels. Mutations of CFTR such as $\Delta$ F508 result in Cystic Fibrosis (CF) causing a wide range of symptoms linked to a defect in fluid transport across the epithelial membranes. The pulmonary symptoms are welldescribed, but the earliest symptoms concern the digestive system. In addition, CF patients are at high risk of developing gastrointestinal cancers (Yamada et al., 2018). The study from Strubberg et al. links for the first time the loss of functional CFTR and gastrointestinal cancer through the increase of the proliferation rate of crypt cells by activating $\mathrm{Wnt} / \beta$-catenin pathway (Strubberg et al., 2018). Indeed, the authors show that loss of functional CFTR leads to an increase of intracellular $\mathrm{pH}(\mathrm{pHi}$ ) levels in crypt cells as $\mathrm{Cl}^{-}$and $\mathrm{HCO}^{-}$secretion is impaired. In addition, it has been shown in Drosophila species that alkaline pHi (negatively charged phospholipids at the inner leaflet) facilitates Dishevelled's interaction with the Wnt receptor Frizzled by addressing Dishevelled to the negatively charged phospholipids through its DEP domain (Simons et al., 2009). Strubberg et al. show that the same mechanism is occurring in Cftr KO cells. The inhibition of CFTR activity alkalinizes pHi in the crypt epithelium, enhancing Dishevelled-2's (Dvl-2) association at the plasma membrane and promoting binding between $\mathrm{Fz}$ and $\mathrm{Dvl}-2$ and therefore facilitating Wnt signaling. Confirming these results, active $\beta$-catenin and LEF1 levels were higher in Cftr KO mice crypts than WT. Furthermore, CFTR has been shown to be a direct target of Wnt signaling as it exhibits an intestine-specific enhancer element positively regulated by TCF4 (Paul et al., 2007). All in all, loss of CFTR facilitates Wnt signaling in the intestinal crypt epithelium, increasing tumorigenesis and underlying the basis of increased risk of gastrointestinal cancers in CF patients.

The main cancer risk for CF patients is gastrointestinal (GI) cancers (Neglia et al., 1995). However, as new therapeutic strategies aim to enhance CF patients' life expectancy, and since the link between loss of functional CFTR and $\mathrm{Wnt} / \beta$ catenin pathway has been assessed in GI cancers, other epithelial cancers could occur in CF patients following the same biological process.

\section{CLCN2}

Adherens junctions and tight junctions ensure epithelium integrity, providing a physical barrier. Adherens junctions further establish the apical-basal cell polarity. The importance of the chloride channel $\mathrm{ClCN} 2$, also called $\mathrm{ClC}-2$, in the regulation of tight junctions' composition in the small intestine has been recently uncovered (Nighot and Blikslager, 2012). As ClCN2 seemed to be an important actor of epithelial integrity and maintenance of the tight junctions, Jin et al. questioned its involvement in adherens junctions (AJ) and its interaction with $\beta$-catenin (Jin et al., 2018). $\beta$-catenin binds E-cadherin in the adherens junctions. If the $\mathrm{AJ}$ are disrupted, $\beta$-catenin is freed from the membrane-bound E-cadherin, accumulates in the cytosol and translocates to the nucleus where it interacts with TCF/LEF1, activating the Wnt target genes.

$\mathrm{ClCN} 2$ is expressed physiologically in the colonic epithelium. Absence of $\mathrm{ClCN} 2$ alters AJ integrity, inducing an increase of nuclear $\beta$-catenin. The increase of nuclear $\beta$-catenin is linked to an upregulation of Wnt target genes. Moreover, the loss of ClCN2 alters colonic crypt homeostasis, increases proliferation and reduces differentiation of colonic cells.

Therefore, the loss of ClCN2 in colonic epithelium leads to the disruption of adherens junction and subsequent activation of Wnt pathway, promoting tumorigenicity. The involvement of other ClCs in the regulation of junctions and their link with Wnt/ $\beta$-catenin pathway has not yet been assessed. However, a study showed that absence of a regulatory protein of Calcium-activated chloride channels (CaCCs) - such as ANO1, also called TMEM16A (Sala-Rabanal et al., 2015; Sala-Rabanal et al., 2017)-CLCA1, induces an increase of $\beta$-catenin in the nucleus (Li et al., 2017). However, this study only focuses on the consequence of a knock-out of CLCA1 and not its link with $\mathrm{CaCC}$ channels nor its direct or indirect interaction with $\mathrm{Wnt} / \beta$ catenin pathway. These points remain to be understood to truly consider CLCA1 as a tumor suppressor via inhibition of Wnt pathway.

\section{WNT SIGNALING PATHWAY INTERPLAY WITH OTHER CHANNELS}

\section{TRP Channels}

Transient receptor protein (TRP) channels are cationic channels that generate $\mathrm{Na}^{+}$and $\mathrm{Ca}^{2+}$ influxes in response to a great variety of stimuli (Venkatachalam and Montell, 2007). Therefore, TRP channels enable individual cells to sense changes in their local environment, translating this information into signals that can be interpreted at a short time scale such as membrane potential and $\mathrm{Ca}^{2+}$ homeostasis (Shapovalov et al., 2016). Several works have revealed a functional link between TRP channels and Wnt/ $\beta$-catenin pathway in cancers.

\section{TRPV4}

In gastric cancers, the Calcium receptor (CasR) is overexpressed. Its overexpression contributes to tumor growth, metastasis formation, and poor prognosis. Mechanistically, CasR stimulates TRPV4 channels. The TRPV4-dependent $\mathrm{Ca}^{2+}$ influx increases AKT activation, which in turn phosphorylates $\beta$-catenin on its residue Ser675 and thus activating $\beta$-catenin downstream signaling (Xie et al., 2017). 


\section{TRPM4}

TRPM4 channels are overexpressed in prostate and colorectal cancers where they participate to migration and invasion (Gao and Liao, 2019). In prostate cancer cells, invalidation of TRPM4 increases GSK3 $\beta$ activity, leading to $\beta$-catenin degradation. In LnCAP prostate cancer cells, TRPM4-associated $\mathrm{Ca}^{2+}$ influx stimulates AKT1, which in turn increases the inhibitory Ser9 phosphorylation of GSK3 $\beta$ and the total amount of $\beta$-catenin (Sagredo et al., 2018). In this study, Sagredo et al. suggest that the effect of TRPM4 on AKT1 is probably mediated by an alteration in the calcium/calmodulin-EGFR axis, linking TRPM4 activity with the observed effects in $\beta$-catenin-related signaling pathways.

\section{TRPM8}

A recent study driven in prostate and breast cancer cell lines has proposed that Wnt ligand-induced signaling involves TRPM8 channels. The authors propose a model in which Wnt ligands bind to both their receptors and TRPM8 channels, inducing a rapid decrease in $\left[\mathrm{Ca}^{2+}\right] \mathrm{i}$, followed by a store-operated calcium entry (SOCE). The SOCE in turn triggers calcium-dependent potassium channels (KCNN4) leading to a large membrane hyperpolarization. This study indeed suggests that control of membrane potential by Wnt ligands is an early event of the Wnt signaling pathway (Ashmore et al., 2019).

\section{TRPC5}

TRPC5 is overexpressed, together with ATP-binding cassette, subfamily 1-member1 (ABCB1) in 5-Fluorouracil (5-FU), in CRC cells. $\mathrm{ABCB} 1$ is a transport protein involved in chemoresistance by mediating efflux of cytotoxic drugs. Enforcing TrpC5 expression increases in a $\mathrm{Ca}^{2+}$-dependent manner nuclear accumulation of $\beta$ catenin and subsequent upregulation of Wnt target genes including $\mathrm{ABCB} 1$. These results suggest that TRPC5 may reinforce chemoresistance by regulating transporters controlled by the Wnt/ $\beta$-catenin pathway (Wang et al., 2015).

\section{Purinergic lonotropic Receptors P2RX7}

The ionotropic ATP receptor P2RX7, (P2X7) is overexpressed in osteocarcinoma and participates to several cancer hallmarks including proliferation, migration, invasion and EMT. At the molecular level, P2X7 stimulation by receptor agonist (bzATP) increases GSK3 $\beta$ phosphorylation and further accumulation of $\beta$-catenin in the nucleus. Indeed, $\mathrm{Wnt} / \beta$-catenin activation by P2X7 stimulation depends on PI3K/AKT stimulation. Interestingly, pharmacological inhibition of P2X7 decreased pro-tumoral behavior of cancer cells (Zhang et al., 2019).

\section{Acid Sensing lon Channels}

ASIC1a: Acid sensing ion channels (Asic) are proton-gated $\mathrm{Na}^{+}$/ $\mathrm{Ca}^{2+}$ channels involved in a wide range of physiological features including pain perception and behavior (Hanukoglu and Hanukoglu, 2016). Asicla is up-regulated in liver cancer tissues and cells lines and is associated to poor prognosis. $\mathrm{KO}$ of Asicla inhibits liver cancer growth in vivo and in vitro, an effect that is underlied by cell cycle arrest and increased apoptosis. Jin et al. observed that low extracellular $\mathrm{pH}$

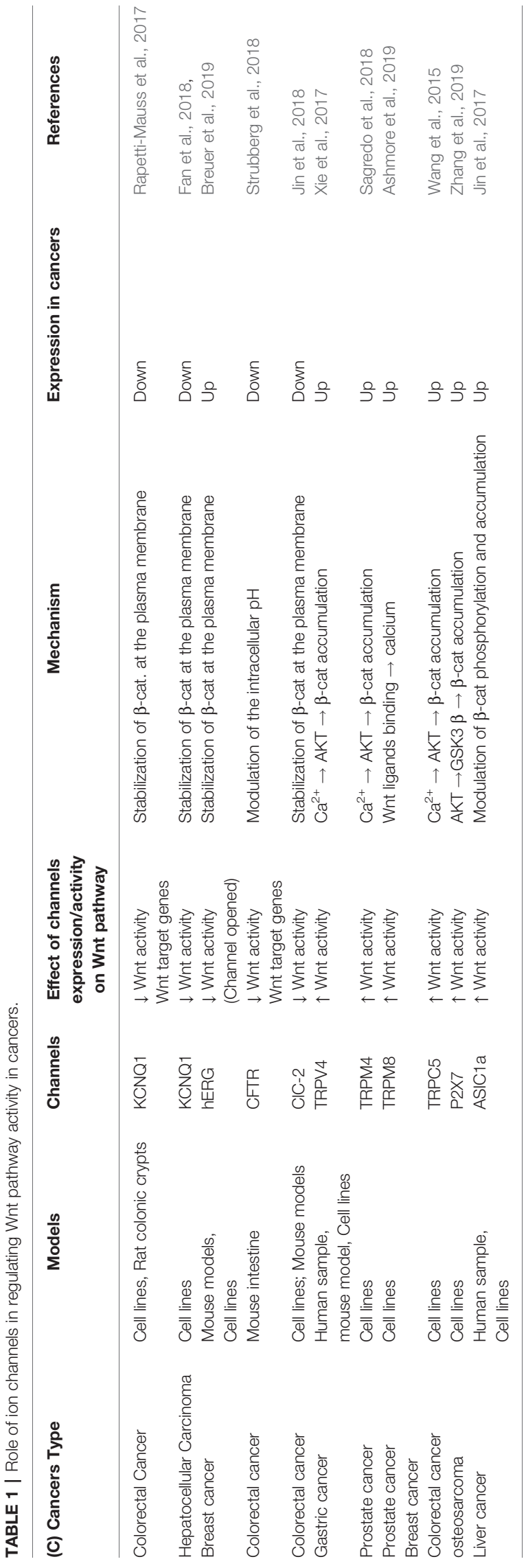



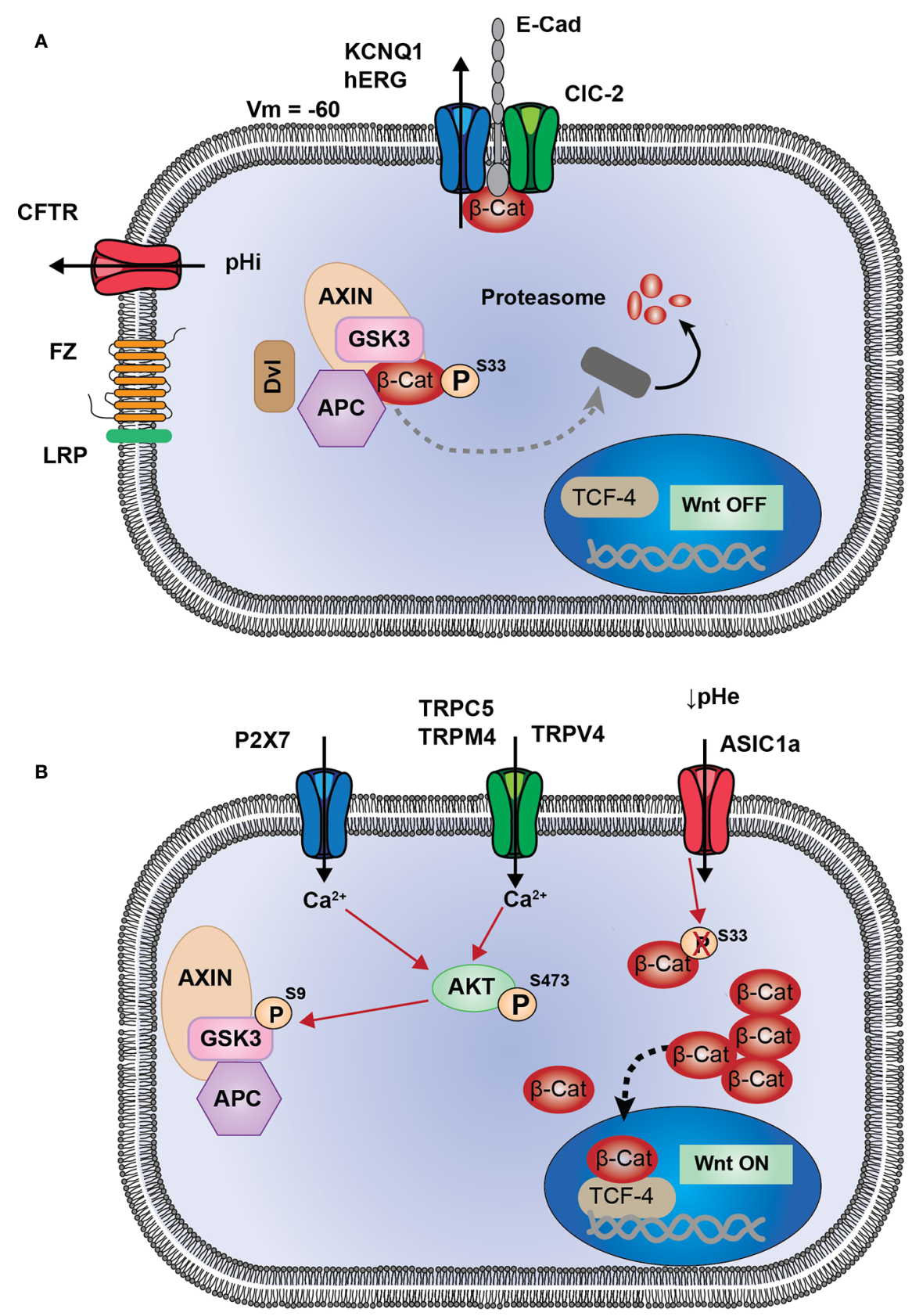

FIGURE 1 | Proposed model of interactions between ion channels and Wnt signaling. (A) KCNQ1 (or hERG) and ClC-2 are associated to E-cadherin and $\beta$-catenin at adherent junctions (AJ). Activity of these two channels maintain membrane potential hyperpolarized, which could be a favorable condition for AJ stability, and sequestration of $\beta$-catenin at the plasma membrane. CFTR activity maintains intracellular $\mathrm{pH}$, preventing from $\mathrm{pHi}$ alkalinisation which induces Dishevelled-dependant inhibition of Destruction Complex (DC). These four channels participate in maintaining Wnt signaling in OFF-state, in which $\beta$-catenin is either degraded or sequestrated and cannot translocate into the nucleus to induce Wnt target genes. (B) $\mathrm{Ca}^{2+}$ entry through TRPC5, TRPM4, TRPV4 and P2X7 channels, induces AKT activation. Thus, AKT phosphorylates GSK3- $\beta$ which becomes inhibited and subsequently allows the cytosolic accumulation of $\beta$-catenin. ASIC1a, by maintaining an acidic $\mathrm{pH}$, decreases $\beta$-catenin phosphorylation and ubiquitination, preventing its degradation and inducing a cytosolic accumulation.

occurring in tumor microenvironment, increased Asicla activity, which in turn decreases the Ser33 phosphorylation of $\beta$-catenin, leading to its accumulation in the nucleus, its combination with LEF/TCF promoting target gene transcription associated to liver cancer proliferation (Jin et al., 2017).

\section{CONCLUSION AND FUTURE DIRECTIONS}

In this review we have summarized the current work regarding the interplay between ion channels and the $\mathrm{Wnt} / \beta$-catenin signaling pathway in cancers. The analysis of the literature 
highlighted 10 ion channels associated to the Wnt pathway (Table 1). Among these channels four are associated to the repression of Wnt pathway (KCNQ1, hERG, CFTR, and ClCN-2, Figure 1A). The loss of activity or expression of these channels leads to an increase of Wnt activity and cancer progression. Supporting this, KCNQ1, CFTR and ClCN-2 have been described as tumor suppressors in CRC. Contrarily, six ion channels are overexpressed in cancers and promote the activity of Wnt pathway (TRPC5, TRPV4, TRPM4, TRPM8, P2RX7, and ASIC1a, Figure 1B).

Several conclusions can be drawn from this analysis of the literature. Firstly, the hyperpolarisation induced by the opening of potassium channel (background current from KCNQ1/ KCNE3 in colonocytes or inducing the opening of hERG in breast cancer cells) leads to the restriction of Wnt activity by a similar mechanism, i.e. the sequestration of $\beta$-catenin at the plasma membrane and probably at the adherens junctions. Hyperpolarised plasma membrane seems to be important to prevent an over activation of the Wnt pathway and stabilise $\beta$ catenin at the plasma membrane. Therefore, the remaining question is: how can membrane potential act on $\beta$-catenin stability at the membrane? This is an outstanding question which will need more investigation to be answered. Secondly, regarding the Wnt-activating ion channels, the mechanism emerging from this analysis seems to mainly involve an increase in intracellular $\left[\mathrm{Ca}^{2+}\right]$ due to the overexpression or up regulation of cationic channels that mediate $\mathrm{Ca}^{2+}$ influx. The increase in intracellular $\left[\mathrm{Ca}^{2+}\right]$ shunts the canonical Wnt/ $\beta$ catenin signaling cascade by activating AKT. AKT, in turn phosphorylates GSK3 $\beta$ on S9 causing its inhibition and finally the accumulation of $\beta$-catenin and the activation of Wnt pathway. Thirdly, as mentioned above, several ion channels are involved in the regulation of Wnt pathway, suggesting that interaction between them could exist. This is true especially regarding the case of KCNQ1, CFTR, and $\mathrm{ClCN}-2$. It is interesting to note that KCNQ1 and $\mathrm{ClCN}-2$ share the same mechanism to lower Wnt pathway activity. Both channels are associated to adherens junctions and contribute to stabilise $\beta$ catenin at the plasma membrane. This observation suggests a functional interaction between these channels which has not been investigated so far. Concerning KCNQ1 and CFTR, it is

\section{REFERENCES}

Abbott, G. W. (2014). Biology of the KCNQ1 Potassium Channel. New J. Sci. 2014, 26. doi: 10.1155/2014/237431

Aberle, H., Bauer, A., Stappert, J., Kispert, A., and Kemler, R. (1997). beta-catenin is a target for the ubiquitin-proteasome pathway. EMBO J. 16, 3797-3804. doi: 10.1093/emboj/16.13.3797

Ashmore, J., Olsen, H., Sørensen, N., Thrasivoulou, C., and Ahmed, A. (2019). Wnts control membrane potential in mammalian cancer cells. J. Physiol. 597, 5899-5914. doi: 10.1113/JP278661

Assié, G., Letouzé, E., Fassnacht, M., Jouinot, A., Luscap, W., Barreau, O., et al. (2014). Integrated genomic characterization of adrenocortical carcinoma. Nat. Genet. 46, 607-612. doi: 10.1038/ng.2953

Azzolin, L., Panciera, T., Soligo, S., Enzo, E., Bicciato, S., Dupont, S., et al. (2014). YAP/TAZ incorporation in the $\beta$-catenin destruction complex orchestrates the Wnt response. Cell 158, 157-170. doi: 10.1016/j.cell.2014.06.013 largely described that in colonic epithelium both channels are closely linked in the mechanism regulating the transepithelial transport of $\mathrm{Cl}^{-}$and water. It seems credible that this functional link is conserved in the regulation of the Wnt pathway, both channels contributing to restrict its activity by two different, but complementary mechanisms, KCNQ1 controlling the stability of $\beta$-catenin at the plasma membrane and CFTR controlling the localization of Dvl. It will be interesting to study the effect of the simultaneous inhibition of both channels on Wnt activity.

Large research efforts have been made during the last 40 years to describe and understand the functions of ion channels in controlling cellular processes. Although the involvement of ion channels in processes participating in cancer development has largely been demonstrated, the precise mechanisms sustaining these abilities remain mainly unknown. Even if several functions of ion channels in signaling pathways can be explained by classical roles, such as regulation of cell volume or modulating the intracellular calcium concentration, other implication of this class of proteins are dictated by original mechanisms. Could ion channels be necessary in the regulation of classical signaling pathway and how? Can we insert ion channels in the notebook picture of signaling pathways beside tyrosine kinases receptors and G protein coupled receptors? Answering these questions are the arising challenges in the field of ion channels in cancer biology.

\section{AUTHOR CONTRIBUTIONS}

RR-M conceived the presented idea and supervised the project. RR-M, CB, BA, and OS wrote the manuscript. All authors contributed to the article and approved the submitted version.

\section{FUNDING}

This work was supported by the Foundation ARC (grant PJA 20181207701), "La Fondation pour la Recherche Médicale"FRM-(PhD studentship to CB) ( ${ }^{\circ}$ ECO201906008972) and the Cancéropole PACA (grant $\mathrm{n}^{\circ}$ 2018-13) and Université Côte d'Azur (CSI-2018).

Bänziger, C., Soldini, D., Schütt, C., Zipperlen, P., Hausmann, G., and Basler, K. (2006). Wntless, a conserved membrane protein dedicated to the secretion of Wnt proteins from signaling cells. Cell 125, 509-522. doi: 10.1016/ j.cell.2006.02.049

Barrett, K. E., and Keely, S. J. (2000). Chloride secretion by the intestinal epithelium: molecular basis and regulatory aspects. Annu. Rev. Physiol. 62, 535-572. doi: 10.1146/annurev.physiol.62.1.535

Becchetti, A., Crescioli, S., Zanieri, F., Petroni, G., Mercatelli, R., Coppola, S., et al. (2017). The conformational state of hERG1 channels determines integrin association, downstream signaling, and cancer progression. Sci. Signal. 10 (473), eaaf3236. doi: 10.1126/scisignal.aaf3236

Becchetti, A., Petroni, G., and Arcangeli, A. (2019). Ion Channel Conformations Regulate Integrin-Dependent Signaling. Trends Cell Biol. 29, 298-307. doi: 10.1016/j.tcb.2018.12.005

Bendahhou, S., Marionneau, C., Haurogne, K., Larroque, M. M., Derand, R., Szuts, V., et al. (2005). In vitro molecular interactions and distribution of KCNE family with 
KCNQ1 in the human heart. Cardiovasc. Res. 67, 529-538. doi: 10.1016/ j.cardiores.2005.02.014

Breuer, E. K., Fukushiro-Lopes, D., Dalheim, A., Burnette, M., Zartman, J., Kaja, S., et al. (2019). Potassium channel activity controls breast cancer metastasis by affecting $\beta$-catenin signaling. Cell Death Dis. 10, 180. doi: 10.1038/s41419-019$1429-0$

Buechling, T., Chaudhary, V., Spirohn, K., Weiss, M., and Boutros, M. (2011). p24 proteins are required for secretion of Wnt ligands. EMBO Rep. 12, 1265-1272. doi: 10.1038/embor.2011.212

Cavallo, R. A., Cox, R. T., Moline, M. M., Roose, J., Polevoy, G. A., Clevers, H., et al. (1998). Drosophila Tcf and Groucho interact to repress Wingless signalling activity. Nature 395, 604-608. doi: 10.1038/26982

Chan-On, W., Nairismägi, M. L., Ong, C. K., Lim, W. K., Dima, S., Pairojkul, C., et al. (2013). Exome sequencing identifies distinct mutational patterns in liver fluke-related and non-infection-related bile duct cancers. Nat. Genet. 45, 14741478. doi: $10.1038 /$ ng.2806

Crottès, D., Rapetti-Mauss, R., Alcaraz-Perez, F., Tichet, M., Gariano, G., Martial, S., et al. (2016). SIGMAR1 Regulates Membrane Electrical Activity in Response to Extracellular Matrix Stimulation to Drive Cancer Cell Invasiveness. Cancer Res. 76, 607-618. doi: 10.1158/0008-5472.CAN-15-1465

Davidson, G., Shen, J., Huang, Y. L., Su, Y., Karaulanov, E., Bartscherer, K., et al. (2009). Cell cycle control of wnt receptor activation. Dev. Cell. 17, 788-799. doi: 10.1016/j.devcel.2009.11.006

de Lau, W., Barker, N., Low, T. Y., Koo, B. K., Li, V. S., Teunissen, H., et al. (2011). Lgr5 homologues associate with Wnt receptors and mediate R-spondin signalling. Nature 476, 293-297. doi: 10.1038/nature10337

DeCoursey, T. E., Chandy, K. G., Gupta, S., and Cahalan, M. D. (1984). Voltagegated K+ channels in human T lymphocytes: a role in mitogenesis? Nature 307, 465-468. doi: 10.1038/307465a0

den Uil, S. H., Coupé, V. M., Linnekamp, J. F., van den Broek, E., Goos, J. A., Delisvan Diemen, P. M., et al. (2016). Loss of KCNQ1 expression in stage II and stage III colon cancer is a strong prognostic factor for disease recurrence. $\mathrm{Br}$. J. Cancer 115, 1565-1574. doi: 10.1038/bjc.2016.376

Fan, H., Zhang, M., and Liu, W. (2018). Hypermethylated KCNQ1 acts as a tumor suppressor in hepatocellular carcinoma. Biochem. Biophys. Res. Commun. 503, 3100-3107. doi: 10.1016/j.bbrc.2018.08.099

Gao, Y., and Liao, P. (2019). TRPM4 channel and cancer. Cancer Lett. 454, 66-69. doi: 10.1016/j.canlet.2019.04.012

Gross, J. C., Chaudhary, V., Bartscherer, K., and Boutros, M. (2012). Active Wnt proteins are secreted on exosomes. Nat. Cell Biol. 14, 1036-1045. doi: 10.1038/ ncb2574

Hanukoglu, I., and Hanukoglu, A. (2016). Epithelial sodium channel (ENaC) family: Phylogeny, structure-function, tissue distribution, and associated inherited diseases. Gene 579, 95-132. doi: 10.1016/j.gene.2015.12.061

Huang, X., and Jan, L. Y. (2014). Targeting potassium channels in cancer. J. Cell Biol. 206, 151-162. doi: 10.1083/jcb.201404136

Huels, D. J., Ridgway, R. A., Radulescu, S., Leushacke, M., Campbell, A. D., Biswas, S., et al. (2015). E-cadherin can limit the transforming properties of activating $\beta$-catenin mutations. EMBO J. 34, 2321-2333. doi: 10.15252/embj.201591739

Jespersen, T., Grunnet, M., and Olesen, S. P. (2005). The KCNQ1 potassium channel: from gene to physiological function. Physiol. (Bethesda) 20, 408-416. doi: 10.1152 /physiol.00031.2005

Jin, C., Yuan, F. L., Gu, Y. L., Li, X., Liu, M. F., Shen, X. M., et al. (2017). Overexpression of ASICla promotes proliferation via activation of the $\beta$-catenin/ LEF-TCF axis and is associated with disease outcome in liver cancer. Oncotarget 8, 25977-25988. doi: 10.18632/oncotarget.10774

Jin, Y., Ibrahim, D., Magness, S. T., and Blikslager, A. T. (2018). Knockout of ClC2 reveals critical functions of adherens junctions in colonic homeostasis and tumorigenicity. Am. J. Physiol. Gastrointest. Liver Physiol. 315, G966-G979. doi: 10.1152/ajpgi.00087.2018

Kadowaki, T., Wilder, E., Klingensmith, J., Zachary, K., and Perrimon, N. (1996). The segment polarity gene porcupine encodes a putative multitransmembrane protein involved in Wingless processing. Genes Dev. 10, 3116-3128. doi: 10.1101/gad.10.24.3116

Kinzler, K. W., Nilbert, M. C., Su, L. K., Vogelstein, B., Bryan, T. M., Levy, D. B., et al. (1991). Identification of FAP locus genes from chromosome 5q21. Science 253, 661-665. doi: 10.1126/science.1651562
Latres, E., Chiaur, D. S., and Pagano, M. (1999). The human F box protein betaTrcp associates with the Cul1/Skp1 complex and regulates the stability of betacatenin. Oncogene 18, 849-854. doi: 10.1038/sj.onc.1202653

Lee, S. C., Deutsch, C., and Beck, W. T. (1988). Comparison of ion channels in multidrug-resistant and -sensitive human leukemic cells. Proc. Natl. Acad. Sci. U. S. A. 85, 2019-2023. doi: 10.1073/pnas.85.6.2019

Li, V. S., Ng, S. S., Boersema, P. J., Low, T. Y., Karthaus, W. R., Gerlach, J. P., et al. (2012). Wnt signaling through inhibition of $\beta$-catenin degradation in an intact Axin1 complex. Cell 149, 1245-1256. doi: 10.1016/j.cell.2012.05.002

Li, X., Hu, W., Zhou, J., Huang, Y., Peng, J., Yuan, Y., et al. (2017). CLCA1 suppresses colorectal cancer aggressiveness via inhibition of the Wnt/betacatenin signaling pathway. Cell Commun. Signal. 15, 38. doi: 10.1186/s12964017-0192-z

Lin, S. Y., Xia, W., Wang, J. C., Kwong, K. Y., Spohn, B., Wen, Y., et al. (2000). Beta-catenin, a novel prognostic marker for breast cancer: its roles in cyclin D1 expression and cancer progression. Proc. Natl. Acad. Sci. U. S. A. 97, 42624266. doi: 10.1073/pnas.060025397

Liu, C., Li, Y., Semenov, M., Han, C., Baeg, G. H., Tan, Y., et al. (2002). Control of beta-catenin phosphorylation/degradation by a dual-kinase mechanism. Cell 108, 837-847. doi: 10.1016/S0092-8674(02)00685-2

Medema, J. P., and Vermeulen, L. (2011). Microenvironmental regulation of stem cells in intestinal homeostasis and cancer. Nature 474, 318-326. doi: 10.1038/ nature 10212

Metcalfe, C., Mendoza-Topaz, C., Mieszczanek, J., and Bienz, M. (2010). Stability elements in the LRP6 cytoplasmic tail confer efficient signalling upon DIXdependent polymerization. J. Cell Sci. 123, 1588-1599. doi: 10.1242/jcs.067546

Mulligan, K. A., Fuerer, C., Ching, W., Fish, M., Willert, K., and Nusse, R. (2012). Secreted Wingless-interacting molecule (Swim) promotes long-range signaling by maintaining Wingless solubility. Proc. Natl. Acad. Sci. U. S. A. 109, 370-377. doi: 10.1073/pnas.1119197109

Neglia, J. P., FitzSimmons, S. C., Maisonneuve, P., Schöni, M. H., Schöni-Affolter, F., Corey, M., et al. (1995). The risk of cancer among patients with cystic fibrosis. Cystic Fibrosis and Cancer Study Group. N. Engl. J. Med. 332, 494-499. doi: 10.1056/NEJM199502233320803

Neumann, S., Coudreuse, D. Y., van der Westhuyzen, D. R., Eckhardt, E. R., Korswagen, H. C., Schmitz, G., et al. (2009). Mammalian Wnt3a is released on lipoprotein particles. Traffic 10, 334-343. doi: 10.1111/j.1600-0854.2008.00872.x

Nighot, P. K., and Blikslager, A. T. (2012). Chloride channel ClC-2 modulates tight junction barrier function via intracellular trafficking of occludin. Am. J. Physiol. Cell Physiol. 302, C178-C187. doi: 10.1152/ajpcell.00072.2011

Nishisho, I., Nakamura, Y., Miyoshi, Y., Miki, Y., Ando, H., Horii, A., et al. (1991). Mutations of chromosome 5q21 genes in FAP and colorectal cancer patients. Science 253, 665-669. doi: 10.1126/science.1651563

Nusse, R., and Clevers, H. (2017). Wnt/ $\beta$-Catenin Signaling, Disease, and Emerging Therapeutic Modalities. Cell 169, 985-999. doi: 10.1016/ j.cell.2017.05.016

Nusse, R., and Varmus, H. E. (1982). Many tumors induced by the mouse mammary tumor virus contain a provirus integrated in the same region of the host genome. Cell 31, 99-109. doi: 10.1016/0092-8674(82)90409-3

Pancrazio, J. J., Viglione, M. P., Tabbara, I. A., and Kim, Y. I. (1989). Voltage-dependent ion channels in small-cell lung cancer cells. Cancer Res. 49, 5901-5906.

Pardo, L. A., and Stühmer, W. (2014). The roles of $\mathrm{K}(+)$ channels in cancer. Nat. Rev. Cancer 14, 39-48. doi: 10.1038/nrc3635

Pastushenko, I., and Blanpain, C. (2019). EMT Transition States during Tumor Progression and Metastasis. Trends Cell Biol. 29, 212-226. doi: 10.1016/ j.tcb.2018.12.001

Paul, T., Li, S., Khurana, S., Leleiko, N. S., and Walsh, M. J. (2007). The epigenetic signature of CFTR expression is co-ordinated via chromatin acetylation through a complex intronic element. Biochem. J. 408, 317-326. doi: 10.1042/BJ20070282

Peifer, M., McCrea, P. D., Green, K. J., Wieschaus, E., and Gumbiner, B. M. (1992). The vertebrate adhesive junction proteins beta-catenin and plakoglobin and the Drosophila segment polarity gene armadillo form a multigene family with similar properties. J. Cell Biol. 118, 681-691. doi: 10.1083/jcb.118.3.681

Preston, P., Wartosch, L., Günzel, D., Fromm, M., Kongsuphol, P., Ousingsawat, J., et al. (2010). Disruption of the K+ channel beta-subunit KCNE3 reveals an important role in intestinal and tracheal Cl- transport. J. Biol. Chem. 285, 7165-7175. doi: 10.1074/jbc.M109.047829 
Prevarskaya, N., Skryma, R., and Shuba, Y. (2010). Ion channels and the hallmarks of cancer. Trends Mol. Med. 16, 107-121. doi: 10.1016/j.molmed.2010.01.005

Prevarskaya, N., Skryma, R., and Shuba, Y. (2018). Ion Channels in Cancer: Are Cancer Hallmarks Oncochannelopathies? Physiol. Rev. 98, 559-621. doi: 10.1152/physrev.00044.2016

Rapetti-Mauss, R., O’Mahony, F., Sepulveda, F. V., Urbach, V., and Harvey, B. J. (2013). Oestrogen promotes KCNQ1 potassium channel endocytosis and postendocytic trafficking in colonic epithelium. J. Physiol. 591, 2813-2831. doi: 10.1113/jphysiol.2013.251678

Rapetti-Mauss, R., Bustos, V., Thomas, W., McBryan, J., Harvey, H., Lajczak, N., et al. (2017). Bidirectional KCNQ1: $\beta$-catenin interaction drives colorectal cancer cell differentiation. Proc. Natl. Acad. Sci. U. S. A. 114, 4159-4164. doi: 10.1073/pnas.1702913114

Rubinfeld, B., Robbins, P., El-Gamil, M., Albert, I., Porfiri, E., and Polakis, P. (1997). Stabilization of beta-catenin by genetic defects in melanoma cell lines. Science 275, 1790-1792. doi: 10.1126/science.275.5307.1790

Sagredo, A. I., Sagredo, E. A., Cappelli, C., Báez, P., Andaur, R. E., Blanco, C., et al. (2018). TRPM4 regulates Akt/GSK3- $\beta$ activity and enhances $\beta$-catenin signaling and cell proliferation in prostate cancer cells. Mol. Oncol. 12, 151165. doi: 10.1002/1878-0261.12100

Sala-Rabanal, M., Yurtsever, Z., Nichols, C. G., and Brett, T. J. (2015). Secreted CLCA1 modulates TMEM16A to activate $\mathrm{Ca}(2+)$-dependent chloride currents in human cells. Elife17 4, e05875. doi: 10.7554/eLife.05875

Sala-Rabanal, M., Yurtsever, Z., Berry, K. N., Nichols, C. G., and Brett, T. J. (2017). Modulation of TMEM16A channel activity by the von Willebrand factor type A (VWA) domain of the calcium-activated chloride channel regulator 1 (CLCA1). J. Biol. Chem. 292, 9164-9174. doi: 10.1074/jbc.M117.788232

Sanchez-Vega, F., Mina, M., Armenia, J., Chatila, W. K., Luna, A., La, K. C., et al. (2018). Oncogenic Signaling Pathways in The Cancer Genome Atlas. Cell 173, 321-337.e310. doi: 10.1016/j.cell.2018.03.035

Satoh, S., Daigo, Y., Furukawa, Y., Kato, T., Miwa, N., Nishiwaki, T., et al. (2000). AXIN1 mutations in hepatocellular carcinomas, and growth suppression in cancer cells by virus-mediated transfer of AXIN1. Nat. Genet. 24, 245-250. doi: $10.1038 / 73448$

Shapovalov, G., Ritaine, A., Skryma, R., and Prevarskaya, N. (2016). Role of TRP ion channels in cancer and tumorigenesis. Semin. Immunopathol. 38, 357-369. doi: 10.1007/s00281-015-0525-1

Sheldahl, L. C., Slusarski, D. C., Pandur, P., Miller, J. R., Kühl, M., and Moon, R. T. (2003). Dishevelled activates Ca2+ flux, PKC, and CamKII in vertebrate embryos. J. Cell Biol. 161, 769-777. doi: 10.1083/jcb.200211094

Simons, M., Gault, W. J., Gotthardt, D., Rohatgi, R., Klein, T. J., Shao, Y., et al. (2009). Electrochemical cues regulate assembly of the Frizzled/Dishevelled complex at the plasma membrane during planar epithelial polarization. Nat. Cell Biol. 11, 286-294. doi: 10.1038/ncb1836

Stamos, J. L., Chu, M. L., Enos, M. D., Shah, N., and Weis, W. I. (2014). Structural basis of GSK-3 inhibition by N-terminal phosphorylation and by the Wnt receptor LRP6. Elife 3, e01998. doi: 10.7554/eLife.01998

Strubberg, A. M., Liu, J., Walker, N. M., Stefanski, C. D., MacLeod, R. J., Magness, S. T., et al. (2018). Cftr Modulates Wnt/ $/$-Catenin Signaling and Stem Cell Proliferation in Murine Intestine. Cell Mol. Gastroenterol. Hepatol. 5, 253-271. doi: 10.1016/j.jcmgh.2017.11.013

Taylor, J. M., and Simpson, R. U. (1992). Inhibition of cancer cell growth by calcium channel antagonists in the athymic mouse. Cancer Res. 52, 2413-2418.

Than, B. L., Goos, J. A., Sarver, A. L., O'Sullivan, M. G., Rod, A., Starr, T. K., et al. (2014). The role of KCNQ1 in mouse and human gastrointestinal cancers. Oncogene 33, 3861-3868. doi: 10.1038/onc.2013.350
Tree, D. R., Shulman, J. M., Rousset, R., Scott, M. P., Gubb, D., and Axelrod, J. D. (2002). Prickle mediates feedback amplification to generate asymmetric planar cell polarity signaling. Cell 109, 371-381. doi: 10.1016/S0092-8674(02)00715-8

Venkatachalam, K., and Montell, C. (2007). TRP channels. Annu. Rev. Biochem. 76, 387-417. doi: 10.1146/annurev.biochem.75.103004.142819

Wang, Y., Krivtsov, A. V., Sinha, A. U., North, T. E., Goessling, W., Feng, Z., et al. (2010). The Wnt/beta-catenin pathway is required for the development of leukemia stem cells in AML. Science 327, 1650-1653. doi: 10.1126/ science. 1186624

Wang, T., Chen, Z., Zhu, Y., Pan, Q., Liu, Y., Qi, X., et al. (2015). Inhibition of transient receptor potential channel 5 reverses 5-Fluorouracil resistance in human colorectal cancer cells. J. Biol. Chem. 290, 448-456. doi: 10.1074/ jbc.M114.590364

Wood, L. D., Parsons, D. W., Jones, S., Lin, J., Sjöblom, T., Leary, R. J., et al. (2007). The genomic landscapes of human breast and colorectal cancers. Science 318, 1108-1113. doi: 10.1126/science. 1145720

Wu, J., Jiao, Y., Dal Molin, M., Maitra, A., de Wilde, R. F., Wood, L. D., et al. (2011). Whole-exome sequencing of neoplastic cysts of the pancreas reveals recurrent mutations in components of ubiquitin-dependent pathways. Proc. Natl. Acad. Sci. U. S. A. 108, 21188-21193. doi: 10.1073/pnas.1118046108

Xie, R., Xu, J., Xiao, Y., Wu, J., Wan, H., Tang, B., et al. (2017). Calcium Promotes Human Gastric Cancer via a Novel Coupling of Calcium-Sensing Receptor and TRPV4 Channel. Cancer Res. 77, 6499-6512. doi: 10.1158/0008-5472.CAN-170360

Yamada, A., Komaki, Y., Komaki, F., Micic, D., Zullow, S., and Sakuraba, A. (2018). Risk of gastrointestinal cancers in patients with cystic fibrosis: a systematic review and meta-analysis. Lancet Oncol. 19, 758-767. doi: 10.1016/S1470-2045(18)30188-8

Yamashita, N., Hamada, H., Tsuruo, T., and Ogata, E. (1987). Enhancement of voltage-gated $\mathrm{Na}+$ channel current associated with multidrug resistance in human leukemia cells. Cancer Res. 47, 3736-3741.

Ye, X., and Weinberg, R. A. (2015). Epithelial-Mesenchymal Plasticity: A Central Regulator of Cancer Progression. Trends Cell Biol. 25, 675-686. doi: 10.1016/ j.tcb.2015.07.012

Zhan, T., Rindtorff, N., and Boutros, M. (2017). Wnt signaling in cancer. Oncogene 36, 1461-1473. doi: 10.1038/onc.2016.304

Zhang, Y., Cheng, H., Li, W., Wu, H., and Yang, Y. (2019). Highly-expressed P2X7 receptor promotes growth and metastasis of human HOS/MNNG osteosarcoma cells via PI3K/Akt/GSK3 $\beta / \beta$-catenin and mTOR/HIF1 $\alpha /$ VEGF signaling. Int. J. Cancer 145, 1068-1082. doi: 10.1002/ijc.32207

Zhou, Y., Wong, C. O., Cho, K. J., van der Hoeven, D., Liang, H., Thakur, D. P., et al. (2015). SIGNAL TRANSDUCTION. Membrane potential modulates plasma membrane phospholipid dynamics and K-Ras signaling. Science 349, 873-876. doi: 10.1126/science.aaa5619

Conflict of Interest: The authors declare that the research was conducted in the absence of any commercial or financial relationships that could be construed as a potential conflict of interest.

Copyright $\odot 2020$ Rapetti-Mauss, Berenguier, Allegrini and Soriani. This is an openaccess article distributed under the terms of the Creative Commons Attribution License (CC BY). The use, distribution or reproduction in other forums is permitted, provided the original author(s) and the copyright owner(s) are credited and that the original publication in this journal is cited, in accordance with accepted academic practice. No use, distribution or reproduction is permitted which does not comply with these terms. 